\title{
A Metamaterial Backed Dipole Antenna for High Gain Directional Communications
}

\author{
Sarin V. P. ${ }^{1}$, Jayakrishnan M. P. ${ }^{2}$, Aanandan C. K. ${ }^{2}$, Pezholil M. ${ }^{2}$ and Kesavath V. \\ ${ }^{1}$ Department of Electronics, Govt. College Chittur, Palakkad, Kerala, India \\ ${ }^{2}$ Centre for Research in Electromagnetics and Antennas, Cochin University of Science and Technology, India \\ E-mail: sarincrema@gmail.com
}

\begin{abstract}
The enhanced radiation performance of a dipole antenna backed by the split ring resonator-continuous wire pair array working in the $\mathrm{H} \perp$ excitation scenario is presented in this paper. The $\mathrm{H} \perp$ excitation scenario of the metamaterial is used to get zero reflection phase resulting in enhanced gain performance. The two layer meta-structure along with the dipole is fabricated on a low cost substrate of dielectric constant 4.4 and height $1 \mathrm{~mm}$. The reflection and transmission characteristics of the metamaterial structure and its effect on the radiation performance of the dipole antenna are presented in this paper.
\end{abstract}

\section{Introduction}

The theoretical invention of Vaselago followed by the practical demonstration of artificial magnetic plasma using an array of split ring resonators (SRR) by J.B Pendry has opened a dramatic change in electromagnetic research field [1-2]. D.R Smith et.al. practically demonstrated the negative refractive index medium by combining the split ring resonator and continuous wire arrays [3]. The peculiar propagation characteristics of negative refractive index materials give rise to different electromagnetic applications like reverse Snell's law, subwavelength focusing etc. Metamaterials has also been used for increasing the radiation performance of antennas. The artificial dielectric property of periodic wire arrays has been used for getting directional radiation patterns from dipole antennas [4]. Kock et.al has used the low refractive index profile of periodic metal plate lens for increasing the directivity of antennas [5]. The near zero refractive index behavior of the metamaterial inclusions gives highly directional radiation from an antenna embedded within such inclusions [6]. The epsilon near zero behavior of the metamaterials can also be used for getting uniform phase distribution over the structure there by shaping the radiation patterns [7].

In conventional Perfect Electric Conductor (PEC) backed dipole antennas, the image currents induced in the ground plane will degrade the impedance matching and radiation performance of the antenna [8]. This disadvantage can be overcome by backing the antenna with High Impedance Surfaces (HIS) [9-11]. The reflection from the HIS structure will be in phase with the incident wave and hence the radiation performance of the antenna can be improved. Another variation includes the Sievenpiper HIS backed dipole antenna in which the radiation pattern can be steered depending upon the Varactor diode bias voltage [12].The degenerate band edge effect offered by photonic crystals give highly directional antennas [13].

In this paper, the directional radiation characteristics offered by a dipole antenna backed by the SRR-wire pair array is proposed. The slow wave right handed resonant mode in the conventional split ring resonator and continuous wire pair array is achieved by utilizing the $\mathrm{H} \perp$ excitation scenario. The antenna shows a front to back ratio of the order of $23 \mathrm{~dB}$, a realized gain of $9 \mathrm{dBi}$ with a very good cross polarization purity and broadside radiation coverage. The simulation studies of the structure is done using the FEM based Ansoft HFSS software and experiments are carried out using PNAE 8362B network analyser.This paper is organized into two sections. The initial section deals with the reflection and transmission characteristics of the SRR-wire pair array in the $\mathrm{H} \perp$ excitation scenario. Section 3 gives the experimental and simulation studies of the SRR-wire pair backed dipole antenna.

\section{The $H_{\perp}$ excitation of $S R R$ and wire pairs}

The metamaterial structure in our design constitutes the standard SRR and wire pair array. D.R Smith proposed two different excitation schemes for this composite [3]. The first one corresponds to the $\mathrm{H}_{\|}$excitation in which the external magnetic field is oriented parallel to the axis of the SRR( perpendicular to the SRR plane) and the E-field is

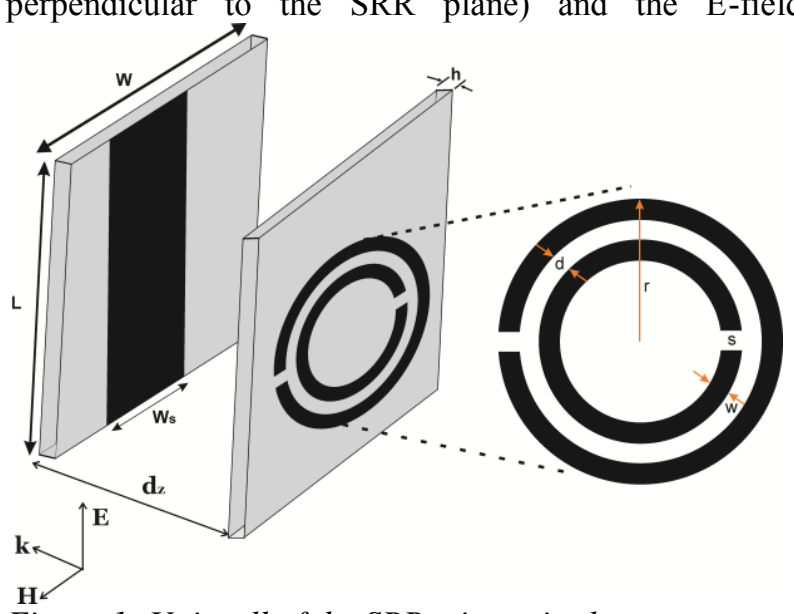

Figure 1: Unit cell of the SRR-wire pair element 
oriented parallel to the splits. This configuration excites the magnetic resonance of the SRR and the resultant medium will be left handed. The second scheme corresponds to the $\mathrm{H} \perp$ excitation, in which the applied magnetic field lies perpendicular to the axis of the SRR (parallel to the plane of the SRR) and the Electric field is oriented parallel to the slit. This gives electric excitation of the SRR and the resultant transmission scenario will be a right handed slow wave resonant mode with reduced group velocity. This pass band occurs when the effective dielectric function of the split ring exceeds that of the wire medium. The magnetic permeability of this medium will be slowly varying. We are adopting this excitation technique for our design. The unit cell of the design is shown in Fig.1. The dimensions of the SRR are $\mathrm{r}=5.2 \mathrm{~mm}, \mathrm{~s}=1 \mathrm{~mm}, \mathrm{w}=1 \mathrm{~mm}$ and $\mathrm{d}=1 \mathrm{~mm}$. The design of the SRR is adopted from the standard design procedure [14]. The design is printed on a low cost substrate of dielectric constant 4.4 and height $1 \mathrm{~mm}$. The unit cell is repeated in an array fashion to form a $13 \times 13$ element array. The width of the continuous wire element is denoted by $\mathrm{w}_{\mathrm{s}}=7 \mathrm{~mm}$.

Unit cell simulation is performed in HFSS to retrieve the reflection magnitude and reflection phase. The $\mathrm{H} \perp$ excitation scenario is achieved in unit cell simulation by assigning Perfect Electric boundary conditions on the top and bottom faces of the unit cell shown in Fig.1 and Perfect Magnetic boundary conditions on the right and left faces of the unit cell. This will ensure the propagation of Transverse Electromagnetic wave with polarization along Y-axis. Waveguide ports are assigned on the front and back face of the unit cell. The resultant reflection coefficient and reflection phase are plotted in Fig.2. The resonant frequency is found to be at $3.23 \mathrm{GHz}$ and the corresponding reflection coefficient is $-10.7 \mathrm{~dB}$. At resonance, the reflection phase goes through zero degrees and the structure effectively acts like an artificial magnetic conductor (AMC). For frequencies lying above and below the resonant frequency, the impedance will be inductive and capacitive respectively. The positive and negative excursions of phase towards $180^{\circ}$ above and below the resonance give PEC behavior for the structure. From the reflection phase studies we can infer that the structure shows two resonances. The lower one is the parallel resonance or the resonant pole and the higher one is the series resonance or called the resonant zero. The parallel resonance is offered by the magnetic resonance offered by the combined effect of SRR and wire pair and it offers impedance equal to infinity at the magnetic resonant frequency. So the reflection phase goes through zero at the resonant frequency. The resonant zero is the series resonance of the structure, in which the series inductance is offered by the inductance of the $S R R$ in $H \perp$ excitation mode and the capacitance is offered by the coupling between adjacent SRR elements in the Y-direction of the array. At the series resonant frequency, the impedance offered by the structure will be zero and reflection phase shows excursions between $\mathbf{- 1 8 0 ^ { \circ }}$ and $+\mathbf{1 8 0}^{\circ}$. This peculiar reflection property makes this structure suitable for enhancing the radiation performance of an antenna around the resonant frequency.

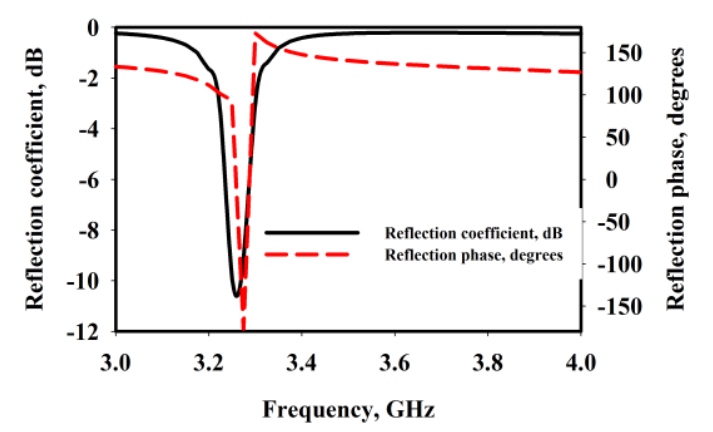

Figure 2: Reflection characteristics of the unit cell

Reflection and transmission measurements on the structure constituting a single array of SRR and continuous wire array have been carried out by adopting the standard procedures. Transmission measurement consists of placing the

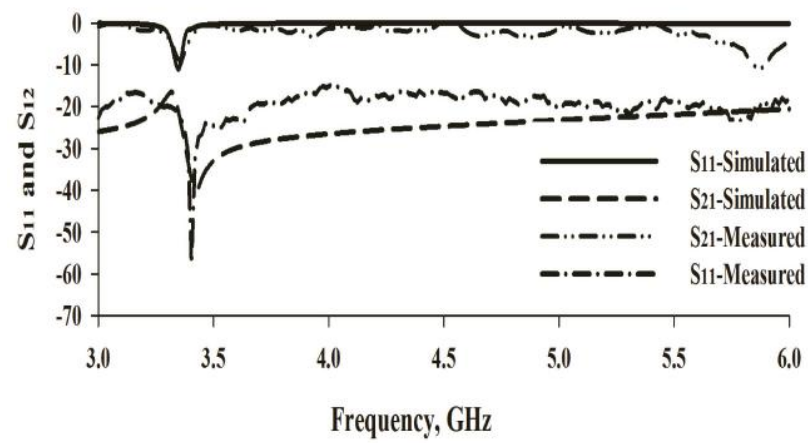

(a)

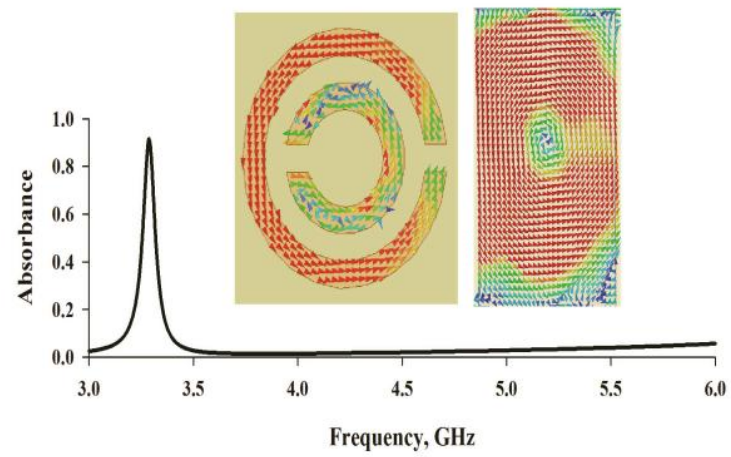

(b)

Figure 3: a) Reflection/Transmission coefficients and b) Absorption coefficient with surface currents on the inset sample in between two ultra wideband horn antennas and measuring the transmission coefficient after the free space TRU calibration. The E-field orientation of the horn antenna is made parallel to the wires at normal incidence for the transmission measurements. Reflection measurements are performed at an angle of $15^{\circ}$ due to practical limitations.

The measured and simulated results are illustrated in fig.3.a. The reflection coefficient attains a value of $-11 \mathrm{~dB}$ at the resonant frequency. It is also noted that the structure shows a very low transmission magnitude of the order of $-21 \mathrm{~dB}$ at the resonant frequency. This means 
that the structure can be utilized as an antireflection coating accompanied by a very low transmission magnitude. The transmission coefficient values lies below $-16 \mathrm{~dB}$ for the entire frequency range in experiment and in simulation. Since the resonance is characterized by a slow wave resonant tunnel band, the decrease in the transmission magnitude is accompanied by the increase in the absorption happening in the structure. The normalized absorbance of the structure is plotted in fig. 3.b. It is observed that the structure shows a normalized absorbance of 0.91 at the resonant frequency. The surface current distributions on the structure corresponding to the resonant frequency are also depicted in the inset of fig.3.b. It is seen that the SRR shows symmetric current distributions on the inner and outer ring yielding the electric dipole response. It is noted that image currents are induced on the wire strip confirming the presence of magnetic resonance constituting the property of artificial Magnetic Conductor and hence the reflection phase goes through zero at the magnetic resonant frequency. Since the resonance is associated with absorption, it can be called as an absorbing Artificial Magnetic conductor ground plane. The resonant zero or the series resonance can be observed from the deep transmission dip in transmission magnitude adjacent to the magnetic resonant frequency.

\section{The Metamaterial Backed Dipole Antenna}

The geometry of the proposed SRR-wire pair backed dipole antenna is shown in Fig.4. The printed half wavelength dipole antenna under consideration is having a dimension of $\mathrm{L}_{\mathrm{d}}=36.5 \mathrm{~mm}$ and $\mathrm{W}_{\mathrm{d}}=5 \mathrm{~mm}$ and is fed using a microstripline to slot line transition for avoiding the radiation due to the unbalanced currents on the feed cable [15]. The dimensions of the balun structure are given by $\mathrm{L}_{1}=18 \mathrm{~mm}, \mathrm{~L}_{2}=10 \mathrm{~mm}, \mathrm{~L}_{3}=8 \mathrm{~mm}$, and $\mathrm{w}=3 \mathrm{~mm}$. The gap

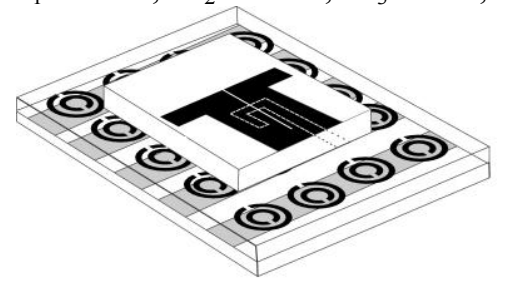

a) Antenna geometry

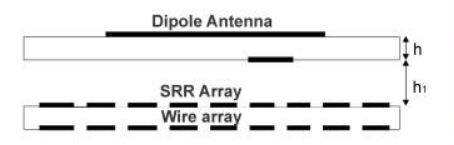

c) Side view

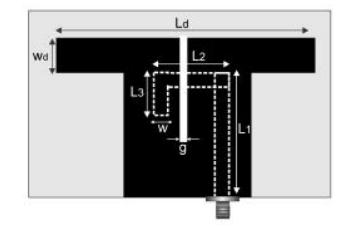

b) Dipole antenna configuration

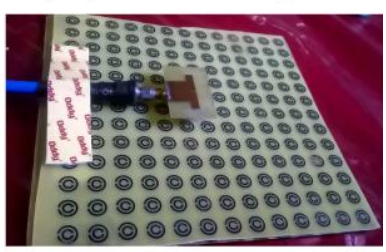

d) Photograph of the antenna
Figure 4: Geometry of the Metamaterial backed dipole antenna

width of the slotline is selected to be $\mathrm{g}=0.5 \mathrm{~mm}$. The SRRwire pair array is loaded on the back side of the dipole antenna and the loading height is denoted by $h_{1}=8 \mathrm{~mm}$ $\left(0.066 \lambda_{0}\right)$. The fabrication of the antenna and the SRR-wire pair arrays are done using the standard photolithographic procedures. The design is fabricated on a low cost epoxy substrate of dielectric constant 4.4 and height $\mathrm{h}=1 \mathrm{~mm}$. For experimental studies, 13X13 element SRR array is backed by the continuous wire elements. The aperture area of the SRR-wire pair array is found to be of order of $1.6 \lambda_{0} X 1.6 \lambda_{0}$. The antenna is fed using the standard Sub Miniaturized Amphenol (SMA) connector soldered at one end of the microstrip line.

Initially, we have studied the performance of the dipole antenna without metamaterial backing. The reflection and radiation characteristics of the antenna are shown in fig.5. The antenna is resonating at $\mathbf{3 . 2 2}$ GHz with a 2:1 VSWR bandwidth of $16.9 \%$. The dipole shows bidirectional radiation patterns in the $H$-plane and figure of eight type pattern in the E-plane. The gain of the antenna is found to be $2.5 \mathrm{dBi}$.

The measured and simulated reflection characteristics of the antenna integrated with the SRR-wire pair array are shown in fig.6. The simulation and experiment are showing fairly good agreement with each other. The close placement of the dipole near the metamaterial layer excites an additional spurious resonance. It is observed that the fundamental dipole resonance is at $3.23 \mathrm{GHz}$ with a $2: 1$ VSWR bandwidth of $5.3 \%$. The reflection coefficient value is found to be $-25.3 \mathrm{~dB}$ at the resonant frequency. The spurious resonance located at $3.52 \mathrm{GHz}$ is having a very low radiation efficiency and gain and hence the study is concentrated on the fundamental mode resonance.

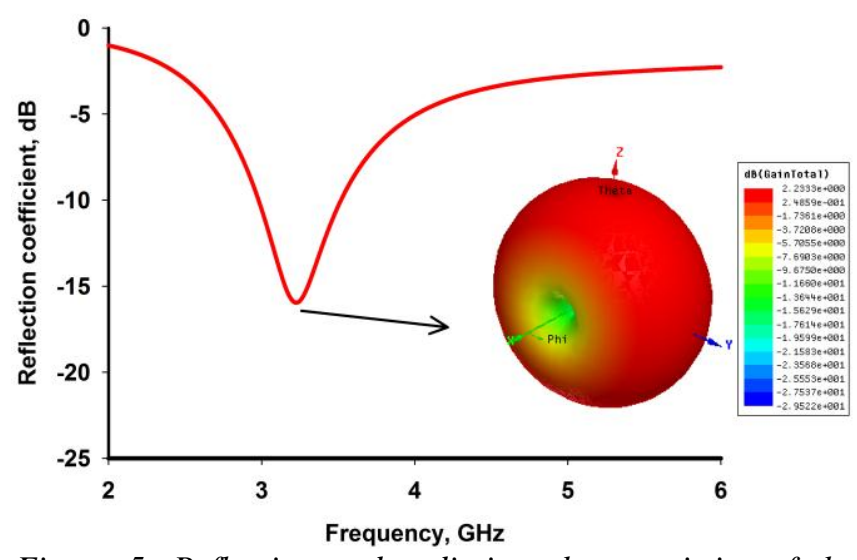

Figure 5: Reflection and radiation characteristics of the dipole antenna

The simulated electric field distributions viewed from the $\theta=0^{0}, \varphi=90^{\circ}$ (in the H-plane) of the HFSS simulation domain is shown in fig.7 (a). It is seen that the SRR-wire pair structure effectively suppresses the back radiation from the dipole antenna and in the near field there is a strong confinement of electric field in between the antenna plane and the AMC ground plane. This will give a high gain directional radiation performance for the antenna. The electromagnetic field enhancement due to the flat tunnel band characteristics in $H \perp$ excitation and the zero reflection phase at the resonant frequency is responsible for the enhanced radiation performance of the antenna. 
The simulated $3 \mathrm{D}$ radiation pattern of the antenna is also shown in fig.7 (b). It is seen that the antenna shows broadside radiation coverage with a front to back ratio in simulations. The patterns show that the radiation in the forward direction is considerably increased as compared to the standard dipole antenna in which the radiation pattern will be omni-directional.

The measured radiation patterns of the antenna in the two principal planes at the resonant frequency are plotted in fig.8. It is seen that the antenna shows a cross polar isolation of $22 \mathrm{~dB}$ in the E-plane. The $3 \mathrm{~dB}$ beamwidth is found to be $51^{0}$ in the E-plane. The H-plane shows a cross polar isolation of $19.6 \mathrm{~dB}$ and the $3 \mathrm{~dB}$ beamwidth is found to be $111^{\circ}$ in that plane. The gain of the antenna is measured using gain comparison method and it is observed that the gain of the antenna is enhanced and is found to be $9 \mathrm{dBi}$. The radiation efficiency of the antenna is measured using the Wheeler Cap method and it is observed that the antenna shows a radiation efficiency of $80 \%$.

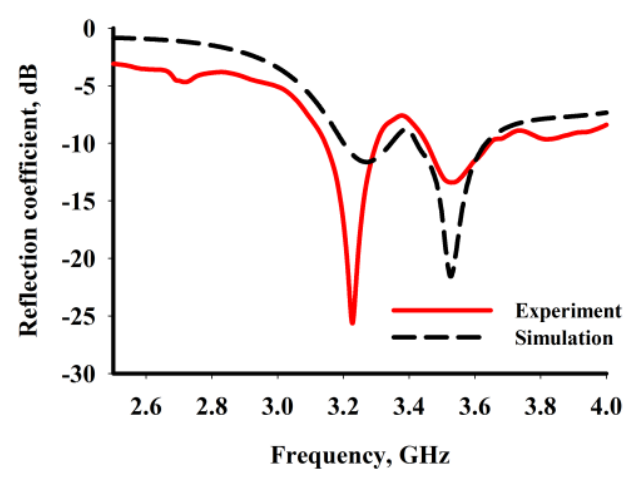

Figure 6: Reflection coefficient of the antenna

Experimental and simulation studies were also performed by varying the size of the metamaterial ground plane. It is observed that the reducing the size of the ground plane affects the peak gain and front to back ratio and the results are summarized in TABLE 1 . It is observed that maximum simulated gain of $8.9 \mathrm{dBi}$ is observed for a $13 \mathrm{X} 13$ element
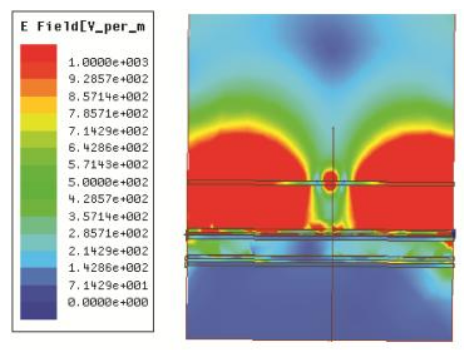

(a)

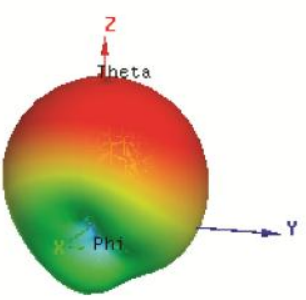

(b)

Figure 7: a) Electric field distributions in the computational domain and b) $3 D$ radiation pattern at the resonant frequency

array. An 8 X8 element array gives a reduced gain of $7.3 \mathrm{dBi}$ with a $\mathrm{F} / \mathrm{B}$ ratio of $20 \mathrm{~dB}$. The area reduced design incorporating a $5 \mathrm{X} 5$ element array gives a much reduced gain of $6.17 \mathrm{dBi}$ and the $\mathrm{F} / \mathrm{B}$ ratio is found to be $18.5 \mathrm{~dB}$.

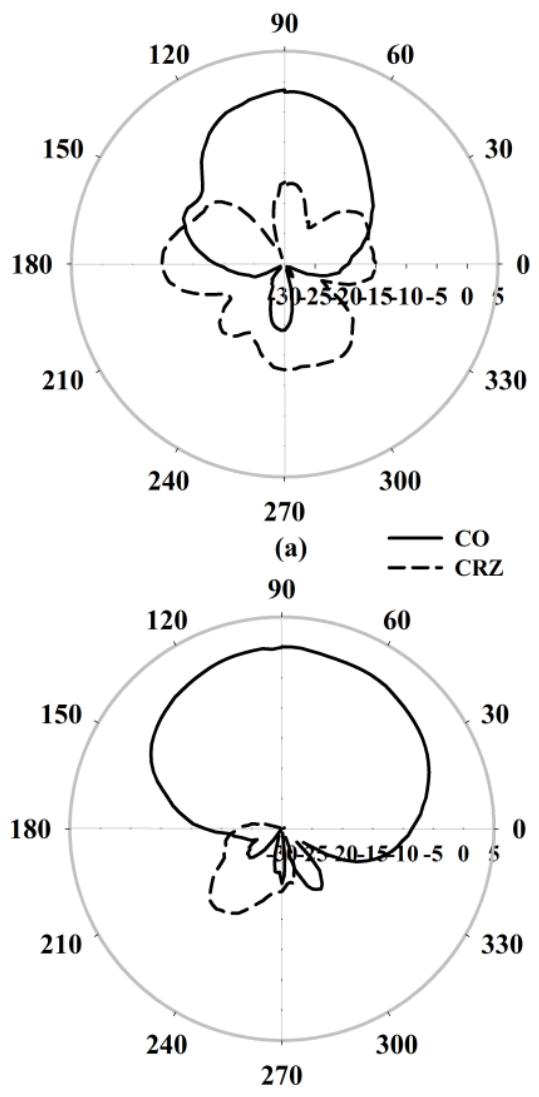

(b)

Figure 8: Measured radiation patterns of the antenna a) Eplane and b) H-plane 
Table 1: Effect of variation in the size of the metamaterial

\begin{tabular}{lccc}
\hline \hline Size & $13 \mathrm{X} 13$ & $8 \mathrm{X} 8$ & $5 \mathrm{X} 5$ \\
\hline $\begin{array}{l}\text { Aperture } \\
\text { area }\end{array}$ & $1.6 \lambda_{0} \mathrm{X} 1.6 \lambda_{0}$ & $1.14 \lambda_{0} \mathrm{X} 1.14 \lambda_{0}$ & $0.68 \lambda_{0} \mathrm{X} 0.68 \lambda_{0}$ \\
$\begin{array}{l}\text { F/B Ratio } \\
\text { Maximum }\end{array}$ & $23 \mathrm{~dB}$ & $20 \mathrm{~dB}$ & $18.5 \mathrm{~dB}$ \\
Gain & $8.9 \mathrm{dBi}$ & $7.3 \mathrm{dBi}$ & $6.17 \mathrm{dBi}$ \\
\hline \hline
\end{tabular}

Inorder to understand the reason behind the second unwanted resonance at $3.52 \mathrm{GHz}$, surface current distributions on the antenna structure have been plotted for the two resonant frequencies. The corresponding diagrams are shown in fig. 9. It is observed that for the fundamental resonance at $\mathbf{3 . 2 3}$ GHz, the dipole arms shows strong in phase current distribution and the SRR elements under the dipole are illuminated well thereby showing directional radiation characteristics at the dipole resonant frequency. At 3.53 GHz, the dipole is not excited and shows very low current density on the dipole arms. Since the resonance is contributed due to the dipolar like resonance offered by the SRR itself, it is having very low radiation efficiency. This is equivalent to the zero resonance of the metamaterial structure.

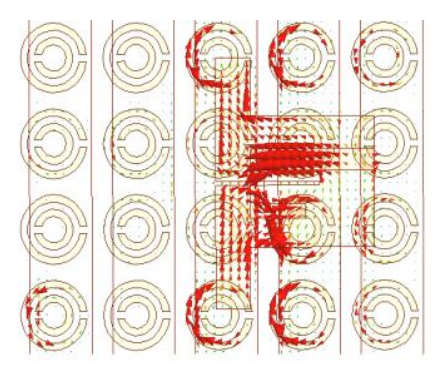

(a)

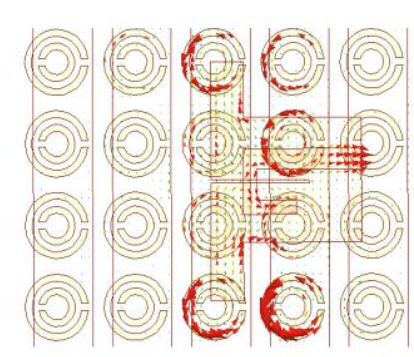

(b)
Figure 9: Simulated surface current distributions on the structure at a) $3.23 \mathrm{GHz}$ and b)3.52 GHz

Parametric analysis has been performed in HFSS for studying the effect of antenna loading height $h_{1}$ on antenna performance. The resultant variation in reflection coefficient of the antenna is plotted in fig.10. It is seen that for the close placement of the antenna above the ground plane $\left(h_{1}=0.022 \lambda_{0}\right)$, impedance matching for the dipole resonance centered around $3.23 \mathrm{GHz}$ is deteriorated. The unwanted second resonance centered at $3.53 \mathrm{GHz}$ is showing maximum matching for this height. The reflection coefficient attains a maximum value of nearly $\mathbf{- 3 0} \mathrm{dB}$ for this resonance. This means that the dipole excitation in SRR will be maximum for this loading height. The radiation efficiency for the second resonance is only $25 \%$. Increase in the loading height increases impedance matching for the fundamental dipole resonance and it inversely affects the second resonance. The loading height of $h_{1}=8 \mathrm{~mm}(0.066$ $\lambda_{0}$ ) is selected in order to reduce the overall volume of the antenna. It is to be noted that for a conventional metal plate backed dipole antenna, the stacking height is of the order

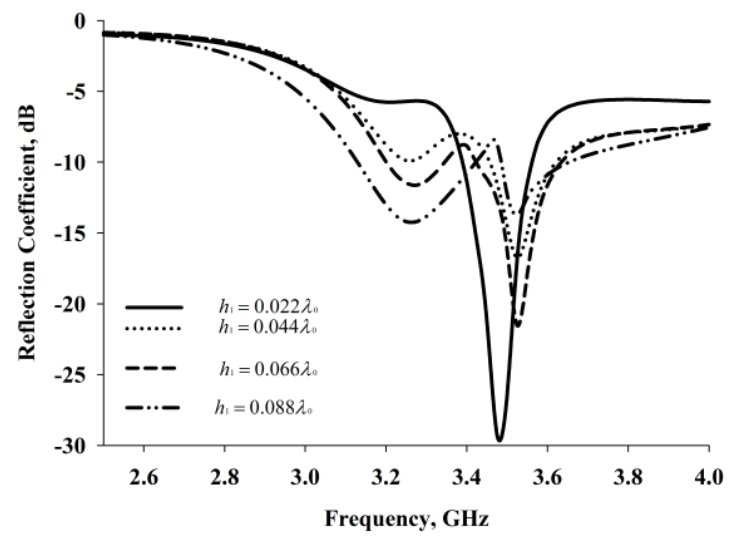

Figure 10: Effect of antenna loading height $h_{1}$ on reflection characteristics

quarter wavelengths corresponding to the dipole resonant frequency, for achieving high gain performance. But here, loading height is very small compared to the conventional metal backed dipole antennas thereby reducing the volume of the structure. In addition, the conventional AMC structures require shorting pins to be soldered between the upper patch and the ground plane [11] or more 3D magnetic inclusions should be stacked [12] which makes more fabrication complexities. But the design we have proposed is fully planar and hence it is devoid of fabrication complexities.

\section{Conclusions}

The SRR-wire pair array backed dipole antenna for improved radiation performance is presented in this paper. Radiation performance enhancement is achieved by utilizing the in phase reflection and electromagnetic field enhancement offered by the metamaterial structure for normal incidence. The $\mathrm{H} \perp$ excitation scenario is utilized to improve the gain up to $9 \mathrm{dBi}$ and the front to back ratio of the directional dipole antenna is found to be $23 \mathrm{~dB}$.

\section{Acknowledgements}

This work was supported in part by the University Grants Commission (UGC), Government of India and Department of Science and Technology (DST), Government of India. 


\section{References}

[1] V.G Vaselago, "The electrodynamics of substances with simultaneously negative values of $\varepsilon$ and $\mu$ ", Soviet Physics Usphekhi, vol. 10, no. 4, pp. 509-514, 1968.

[2] J. B. Pendry, A. J. Holden, D. J. Robbins, andW. J. Stewart, "Magnetism from conductors and enhanced nonlinear phenomena," IEEE Trans. Microwave Theory Tech, vol. 47, pp. 2075-2081, 1999.

[3] D. R. Smith, W. J. Padilla, D. C. Vier, S. C. NematNasser, and S. Schultz, "Composite medium with simultaneously negative permeability and permittivity," Phys. Rev. Lett., vol. 84, no. 18, pp.4184-4187, 2000.

[4] K.C Gupta, "Narrow beam antennas using an artificial dielectric medium with permittivity less than unity", IET Electronics Lett., vol.7, no.1, pp. no.16-18, 1970.

[5] Winston Kock, "Metal-Lens antennas", Proceedings of I.R.E and Waves sand Electrons, pp. no. 828-836, 1946.

[6] Zhi Hao Jiang, Qi Wu, Douglas H. Werner “ Demonstration of enhanced broadband unidirectional electromagnetic radiation enabled by a subwavelength profile leaky anisotropic zero index metamaterial coating", Physical review B, 86, 125131, 2012.

[7] Andrea Alu, Mario Silverihna, Alessandro Salandrino, Nader Engheta, "Epsilon near zero metamaterials and electromagnetic sorces: Tailoring the radiation phase pattern", Physical review B, 75, 155410, 2007.

[8] Mohammed Ziaul Azad, Mohammod Ali, "Novel wideband directional dipole antenna on a mushroom like EBG structure," IEEE Tans. Antennas and Propag., vol. 56, no. 5, pp.1242-1250, 2008.

[9] Andrea Vallecchi, Javier R. De Luis, Filippo Capolino, Fransco De Flaviis, "Low profile fully planar folded dipole antenna on a high impedance surface", IEEE Tans. Antennas and Propag., vol. 60, no. 1, pp.51-62, 2012.

[10] Sangkil Kim, Yu-Jiun Ren, Heseon Lee, Symeon Nikolaou, Manos M. Tentzeris, "Monopole antenna with inkjet printed EBG array on paper substrate for wearable applications", IEEE Antennas and wireless propag. Lett, vol. 11, pp.663-666, 2012.

[11] Aycan Erentok, Paul L. Luljack, Richard W. Ziolkowski, "Characterisation of a volumetric metamaterial realization of an artificial magnetic conductor for antenna applications", IEEE Tans. Antennas and Propag., vol. 53, no. 1, pp.160-172, 2005.

[12] Dan Sievenpiper, Jim Schaffner, J.J Lee, Stan Livingston, "A steerable leaky wave antenna using a tunable impedance ground plane", IEEE Antennas and wireless propag. Lett, vol. 1, pp.179-182, 2002.

[13] Salih Yarga, Kubilay Sertel, John L. Volakis, "Degenerate band edge crystals for directive antennas", IEEE Tans. Antennas and Propag., vol. 56, no. 1, pp.119-126, 2008.

[14] Anju Pradeep, Mridula S, P. Mohanan, "Design of an edge coupled dual ring split ring resonator", IEEE Antennas and Propag. Mag.,vol. 53, no.4, pp. no. 45-54, 2011.
[15] D. Jaisson, "Fast design of a printed dipole antenna with an integrated balun",IEE Proc. Micow. Antennas Propag., vol.153, no.4, pp.no. 389-394, 2006. 